Projets

de paysage

\section{Projets de paysage}

Revue scientifique sur la conception et l'aménagement de l'espace

9 | 2013

Le paysage a t-il imposé sa loi ?

\title{
Analyse interprétative du discours du Landscape Urbanism et impact sur le développement des pratiques des paysagistes dans le contexte français
}

Discourse Analysis of Landscape Urbanism and its impact on the development of Landscape Architects' professional practices in the French context

\section{Anaïs Leger-Smith}

\section{(Q) OpenEdition}

\section{Journals}

Édition électronique

URL : http://journals.openedition.org/paysage/12682

DOI : $10.4000 /$ paysage. 12682

ISSN : 1969-6124

Éditeur :

École nationale supérieure du paysage de Versailles-Marseille, Institut national des sciences appliquées Centre Val de Loire - École de la nature et du paysage, École nationale supérieure d'architecture et de paysage de Bordeaux, École nationale supérieure d'architecture et de paysage de Lille, Agrocampus Angers

Référence électronique

Anaïs Leger-Smith, « Analyse interprétative du discours du Landscape Urbanism et impact sur le développement des pratiques des paysagistes dans le contexte français », Projets de paysage [En ligne], 9 | 2013, mis en ligne le 20 décembre 2013, consulté le 18 novembre 2020. URL : http:// journals.openedition.org/paysage/12682 ; DOI : https://doi.org/10.4000/paysage.12682

Ce document a été généré automatiquement le 18 novembre 2020.

Projets de paysage 


\section{Analyse interprétative du discours du Landscape Urbanism et impact sur le développement des pratiques des paysagistes dans le contexte français}

Discourse Analysis of Landscape Urbanism and its impact on the development of Landscape Architects' professional practices in the French context

\section{Anaïs Leger-Smith}

Depuis les années 2000, la proportion de la population vivant dans des espaces urbains est majoritaire : nous sommes entrés dans «le premier siècle urbain » (Steiner, 2011). Le rythme rapide d'urbanisation soumet les écosystèmes à l'influence des villes et des environnements construits. Le développement durable est présenté comme une réponse, bien que certains auteurs jugent le concept insuffisant (Marcuse, 1998) et préfèrent affirmer la nécessité d'un réel «tournant écologique » (Reimer, 2010) dans la manière de penser stratégiquement les espaces urbains. Ce tournant se traduit aujourd'hui par l'émergence de nouvelles théories et pratiques professionnelles en urbanisme. Cela concerne aussi les paysagistes dont le domaine d'expertise est traditionnellement positionné à l'interface entre architecture et sciences naturelles. Les implications théoriques de ce tournant écologique s'expriment dans les discours paysagistes.

2 Charles Waldheim a développé la théorie du Landscape Urbanism aux États-Unis au début des années 2000 pour proposer « un réalignement disciplinaire dans lequel le paysage supplante le rôle traditionnel de l'architecture comme unité de construction du projet urbain» (Waldheim, 2006b). Le Landscape Urbanism constitue aujourd'hui l'une des théorisations les mieux formulées et les plus pertinentes reliant la notion de paysage aux sciences écologiques. Cet article est une analyse interprétative du discours professionnel du Landscape Urbanism, et de son impact sur la profession de paysagiste et 
l'évolution de ses pratiques, notamment dans le contexte français. Le Landscape Urbanism est-il un discours professionnel de promotion du paysagisme dans un contexte concurrentiel vis-à-vis des autres professionnels de la conception urbaine ou reflète-t-il une réelle évolution des pratiques paysagistes au regard des nouveaux enjeux écologiques urbains?

Dans la première section, nous précisons le cadre théorique de notre recherche et examinons l'évolution de la relation entre paysage et écologie dans les sciences du paysage ainsi que le rôle du discours professionnel comme facteur d'évolution des pratiques paysagistes. Nous établissons alors nos hypothèses de recherche et notre méthodologie. Une deuxième et troisième sections présentent une analyse interprétative du discours du Landscape Urbanism en parallèle avec le discours de trois paysagistes français et la théorie du paysage en France. Finalement, la dernière partie aborde des perspectives de réflexion sur le lien entre le Landscape Urbanism et les domaines de la recherche et de l'enseignement.

\section{Évolution des pratiques et des discours paysagistes au regard des nouveaux enjeux écologiques}

\section{Le paysage urbain, un médium pour le développement durable urbain}

4 Le rapport Brundtland (1987) a introduit la notion de développement durable comme « un développement qui répond aux besoins du présent sans compromettre la possibilité pour les générations à venir de satisfaire les leurs ». Le concept de développement durable urbain traduit donc la nécessité de lier les processus économiques et sociaux aux problèmes environnementaux locaux, régionaux et globaux, dans un contexte d'augmentation de la population dans les espaces urbains et tente de les allier avec une construction écologique (Repetti et Desthieux, 2006). Dans ce contexte, le concept de paysage urbain est formulé comme moyen opérationnel et médium pour les questions d'aménagement urbain (Jannière et Pousin, 2007). Lindholm (2011) confirme cette idée en énonçant que «le concept de paysage est adapté aux modes de pensée interdisciplinaires et dialogiques du développement durable». Cependant, la traduction opérationnelle des principes du développement durable est difficile à identifier et à mettre en œuvre, spécialement dans le domaine de l'aménagement urbain. Cette notion provoque le scepticisme de certains théoriciens qui reprochent l'imprécision du concept et dénoncent un "slogan manipulateur et confus » (Eisen, 1999) ou un « outil de marketing territorial ( (Emelianoff, 2004). Aussi, certains auteurs suggèrent que l'approche écologique puisse prendre le dessus sur l'approche durable dans le domaine de l'aménagement urbain (Corner, 2006; Nassauer, 2012; Waldheim, 2006a). 


\section{Quelques jalons historiques pour saisir l'évolution de la relation entre paysagisme et écologie : vers une formulation du paysage comme mode opératoire de la conception urbaine écologique}

$5 \quad$ Historiquement, l'écologie était intégrée aux réflexions et aux pratiques paysagistes dès la deuxième moitié du xix ${ }^{e}$ siècle aux États-Unis, en particulier chez Frederick Law Olmsted. Son «système de parcs » proposait une offre de nature urbaine de proximité, en complément et opposition à la nature distante et sauvage du wilderness (Cormier, 2011). Les façons d'articuler les questions de paysage et d'écologie ont ensuite cherché à répondre aux nouveaux défis environnementaux de l'aménagement mis en évidence dans la deuxième moitié $\mathrm{du} \mathrm{xx}^{\mathrm{e}}$ siècle. Après les années 1960 et leur "révolution environnementale » (Swaffield, 2002), McHarg (1969) conteste les principes esthétiques de la conception pure dans son ouvrage Design with Nature et développe une approche sous le terme de "Ecological Method ». Cet ouvrage constitue un des points de départ des pratiques écologiques en conception urbaine et paysagère. En 1970, le modèle compartimenté d'Odum (2005) établit un nouveau modèle écologique intégrant à part entière les activités humaines, en contraste avec les approches biocentriques des écologues.

6 La recherche en écologie s'intéresse alors progressivement au contexte urbain. Le champ de l'écologie urbaine est «l'étude scientifique des processus déterminant l'abondance et la distribution des organismes, l'interaction entre les organismes, l'interaction entre les organismes et l'environnement, et les flux d'énergie et de matériaux à travers les écosystèmes, dans les systèmes urbains » (Gaston, 2010). Il s'agit donc de l'écosystème urbain liant l'homme et la nature (Alberti, 2008). L'association entre les notions de paysage et d'écologie s'est plus récemment manifestée à travers l'écologie du paysage (Forman et Gordon, 1986). Celle-ci introduit la prise en compte explicite de la dimension spatiale des processus écologiques "à l'échelle du paysage ", renforçant les interactions entre écologues et aménageurs. Cette discipline est utilisée comme base théorique pour les études paysagères mais aussi comme outil de planification urbaine et régionale. Ainsi, les concepts scientifiques «corridors, matrices, taches" sont transférés dans l'action publique (Cormier, 2011). Snep et Opdam (2010) mettent en évidence à l'échelle du paysage urbain un réseau d'écosystèmes urbains ancré dans la matrice urbaine. Ils considèrent les écosystèmes, éléments verts dans le paysage urbain, comme une part intrinsèque du système urbain.

7 L'écologie urbaine ou l'écologie du paysage constituent aujourd'hui un corpus scientifique sur lequel s'appuient les principes opérationnels de conception urbaine de certains paysagistes. Cependant, l'enjeu pratique reste de mieux relier l'expérience visuelle du paysage aux processus environnementaux (Nassauer, 2012). Nassauer en tire deux «principes » : le paysage est un médium, un catalyseur de synthèse scientifique et il constitue une méthode de conception qui invite à la créativité. Plus pratiquement, le paysage en tant que médium peut contribuer au savoir scientifique et le rendre plus applicable à la conception urbaine écologique. Ainsi, le paysage rend l'écologie plus opérationnelle. Certains nouveaux concepts opérationnels d'urbanisme verts comme par exemple écocities, sustainable cities et green cities, combinent la conception urbaine « traditionnelle » avec une certaine attention au paysage et aux systèmes écologiques.

8 En France, malgré l'essor de l'écologie du paysage (Burel et Baudry, 1999), la dichotomie persiste entre les domaines du paysage et de l'écologie dans la mesure où la 
«théorie du paysage » (Roger, 1997) a été construite sur la distinction du pays et du paysage et, en arrière-plan, sur l'opposition entre nature et culture à laquelle elle renvoie (Descola, 2005). Or, Berque (2000) propose une définition, partagée par Roger, du milieu comme jouant un rôle pivot à l'interface entre l'environnement dans sa dimension physique et le paysage dans sa dimension sensible entre lesquels existe une relation ambivalente ou trajection. La double perception, à la fois objective et subjective de ce processus, constituerait la médiance. Aussi, au lieu de l'opposition entre technique et nature, inhumanité et humanité, Latour (1999) recommande un gouvernement conjoint des hommes et de la nature, sans distinction entre le naturel et l'artificiel (Deleuze et Guattari, 1980 ; Donadieu et Périgord, 2005). Cette dissociation théorique entre paysage et écologie a des effets sur les pratiques paysagistes françaises et sur la manière dont les paysagistes introduisent des notions écologiques dans leur travail en France.

9 Le projet de paysage se place donc aujourd'hui comme mode d'opérationnalisation privilégié des principes des sciences écologiques. Le Landscape Urbanism s'inscrit dans cette ligne théorique.

\section{Théories et discours comme facteurs d'évolution des pratiques paysagistes}

10 Depuis vingt-cinq ans, un débat a lieu en paysage sur le domaine de connaissance de cette discipline ainsi que sur la définition et le statut d'une théorie en paysage. En premier lieu, nous nous intéressons à la disciplinarité en tant que spécialité universitaire, constituant une démarcation entre domaines de connaissances définis par leur méthodologie de recherche et leur jargon (Fry, 2001). Une discipline autonome est donc basée sur un corps de connaissances spécialisé. Selon Deming et Swaffield (2011), le savoir explicite définit la discipline à travers les publications universitaires, l'enseignement et les discours professionnels formels. Le savoir systématique se situe dans la recherche, les revues et les thèses. Le savoir opérationnel est défini par les compétences professionnelles requises pour l'accréditation par un corps professionnel par exemple. Notre article s'attache particulièrement aux discours produits par les professionnels du paysage. Ces discours émanent de préoccupations théoriques et pratiques communes à la discipline du paysage, traitant par exemple des valeurs, ou des problèmes pratiques de conception ou de mise en oeuvre. Certains de ces discours peuvent faire office de modèles pour le reste de la profession.

11 Une théorie peut se révéler être un facteur de stabilité pour la profession, lorsqu'elle souligne les codes et la connaissance opérationnelle de la discipline, ou de perturbation, lorsqu'elle remet en question la nature des pratiques et provoque un changement (Corner, 1990). Si une théorie est définie comme une « relation plausible entre concepts » (Silverman, 2005, p. 98), elle peut se baser, d'une part, sur un modèle scientifique positiviste et des données factuelles ou, d'autre part, sur « une approche interprétative, basée sur un contexte particulier " (Corner, 1990). Ainsi, une théorie instrumentale décrit et contrôle les actions pratiques, une théorie critique stimule le changement et une théorie interprétative s'appuie sur l'analyse du contexte (Swaffield, 2002). Chaque discours est propre à la démarche de son auteur, impactant la nature de la théorie produite. Appliqué au domaine des pratiques de conception en paysage, le 
statut d'une théorie peut s'avérer ambigu. Comment différencier une théorie d'un « nouveau mouvement de conception à la mode » (Corner, 1990)?

\section{Questions, hypothèses de recherche et méthodologie}

Nous observons aujourd'hui un développement du discours des paysagistes sur des thématiques écologiques. Le Landscape Urbanism est aujourd'hui un des discours dominants traitant des questions écologiques en paysage, avec la publication de l'ouvrage central Landscape Urbanism Reader en 2000, d'articles sur le sujet dans Journal of Landscape Architecture JoLA (Donadieu, 2006) ou Landscape Research (Thompson, 2012), ainsi que dans les numéros spéciaux des revues Topos (numéro 71) et Anthos (mai 2013). C'est dans ce cadre que nous cherchons à analyser la nature et le statut du Landscape Urbanism en tant que théorie du paysage. Nous examinons le rôle que celle-là peut jouer dans l'évolution des pratiques paysagistes de conception ainsi que les similarités avec d'autres théories, en particulier en France. L'objectif principal de cet article et de comprendre si le Landscape Urbanism constitue un facteur d'évolution, de renouvellement et d'innovation des pratiques paysagistes de conception.

Nous formulons trois hypothèses de recherche :

1. cette théorie constitue un discours professionnel exploitant l'actuel tournant écologique en urbanisme pour promouvoir les professions du paysage ;

2. bien que ce discours soit promotionnel, il constitue néanmoins un référentiel pour les pratiques paysagistes contemporaines;

3. les discours professionnels comme le Landscape Urbanism influencent les autres mouvements de théorisation et de recherche en paysage, et contribuent au renforcement de l'identité disciplinaire du paysage et de sa pédagogie.

Notre méthodologie se place dans la lignée des stratégies interprétatives : l'analyse de discours (Deming et Swaffield, 2011). Cela implique une interprétation subjective des mots et des textes. Le chercheur devient un acteur social de son investigation, mais doit pourtant conserver une posture distanciée. L'objectif de cette méthodologie est d'étudier le contenu et le sens du discours dans un contexte particulier, au sein d'une communauté professionnelle. Il analyse «la structure du langage à travers les textes, ainsi que le contexte social et culturel au sein duquel il est produit» (Paltridge, 2007). Cette analyse peut, d'une part, constituer un instrument objectif, focalisé sur l'identification du contenu et d'aspects dominants et, d'autre part, adopter une perspective plus critique pour « révéler des valeurs et des positions dissimulées dans le discours" (ibid.). Empruntant au domaine de la géographie sociale, l'analyse du discours cherche à « expliquer les pratiques des individus, des groupes et les principes de régulation qui les organisent, influencés par différents vecteurs, comme les situations sociales contextualisées, ou bien les différentes formes de langage " (Bertrand et al., 2007). Dans le domaine de la linguistique, l'analyste du discours s'interroge sur le genre de discours lui-même, sur la composition textuelle, sur les rôles sociodiscursifs qu'il implique (Maingueneau, 2005). L'analyse du discours est donc présentée comme «l'analyse du langage utilisé » mais aussi comme « une investigation de ce pour quoi le langage est utilisé » (Brown et Yule, 1983). En outre, l'argumentation est analysée comme faisant partie du fonctionnement discursif au même titre que les autres processus langagiers (Amossy, 2008) : cela va de la confrontation explicite des thèses à la coconstruction d'une réponse à une question donnée et à l'expression 
spontanée d'un point de vue personnel. Pour d'autres cependant, l'argumentation constitue un fait de langue et non de discours et ne relève pas de l'art de persuader (Ducrot, 2004). Ainsi, «l'argumentation rhétorique " s'oppose à «l'argumentation linguistique ». Il faut donc étudier de près la façon dont l'argumentation s'inscrit, non seulement dans la matérialité discursive mais aussi dans l'interdiscours.

En paysage, deux méthodologies d'analyse de discours sont utilisées: l'analyse de sources directes et l'analyse de sources secondaires. La première se base sur des entretiens. Un exemple important en paysage utilisant cette méthodologie est le travail de Ian Thompson de l'université de Newcastle au Royaume-Uni. Il a réalisé en 2000 une série d'entretiens de paysagistes praticiens britanniques afin de comprendre les valeurs renforçant la profession. L'analyse de sources secondaires se base sur des discours écrits. Il existe peu d'exemples de travaux de recherche de ce type. Ian Thompson (2012) a récemment publié un article dans Landscape Research sur l'analyse du discours du Landscape Urbanism, dans lequel il réalise une "interprétation» et même une « analyse critique » de ce discours.

Dans cet article, notre méthodologie est une analyse interprétative de discours, examinant des textes, des théories et des discours émanant de la profession. L'article met en parallèle les idées mobilisées dans l'ouvrage fondateur du Landscape Urbanism Reader, mené essentiellement par l'architecte Charles Waldheim et le paysagiste James Corner, avec les textes centraux de trois paysagistes français : Michel Desvigne, Gilles Clément et François-Xavier Mousquet, tirés de monographies ou d'articles de recherche, ainsi que la théorie du paysage émanant de la recherche en paysage en France, le projet de paysage. Il examine aussi différents articles de recherche internationaux émanant notamment du Conseil européen des écoles de paysage (European Council of Landscape Architecture Schools) ou de revues de recherche telles que Landscape Research. Nous adoptons une perspective interprétative subjective. Ce travail fait partie d'un projet de thèse qui implique aussi des entretiens avec une dizaine de paysagistes et une dizaine de chercheurs.

\section{Le Landscape Urbanism : du discours aux pratiques, une opportunité pour les paysagistes}

En l'absence de "critiques substantielles » sur le mouvement du Landscape Urbanism (Thompson, 2012, p. 8), les paragraphes suivants se proposent d'aborder les tensions entre nouveaux discours professionnels, théories académiques et réelle émergence de pratiques professionnelles.

\section{Le Landscape Urbanism : un discours professionnel de paysagistes pour occuper la nouvelle niche écologique}

18 Le Landscape Urbanism a été créé par une lignée de praticiens universitaires américains. Ian McHarg, "grand-père » du mouvement, d'origine écossaise et émigré dans les années 1950 aux États-Unis, en a véritablement posé les bases à travers son ouvrage Design with nature (1969). Le " père ", Charles Waldheim, est un architecte américain qui fut l'élève de McHarg et est aujourd'hui le directeur du département Paysage de la Harvard University Graduate School of Design. Il a formulé le premier le terme de 
Landscape Urbanism et est l'éditeur de l'ouvrage central Landscape Urbanism Reader. Enfin, James Corner, paysagiste américain à New York, fut l'élève de McHarg à la University of Pennsylvania School of Design où il est directeur du département Paysage et recrute la plupart de ses collaborateurs pour travailler dans son agence new-yorkaise Field Operations. Il a contribué en grande partie au Landscape Urbanism Reader. Ce discours est donc formulé en majorité par des praticiens opérationnels américains, devenus professeurs.

En énonçant que le paysage supplante le rôle traditionnel de l'architecture comme unité de construction du projet urbain, Waldheim (2006b) définit le Landscape Urbanism comme "réalignement disciplinaire ", promouvant le paysagisme comme la pratique professionnelle la plus appropriée. Selon Steiner (2011), praticien et universitaire américain, le mouvement intègre « le plaidoyer écologique de McHarg » avec la « vision de la conception urbaine de Corner ». Le Landscape Urbanism défie la dichotomie nature/culture et encourage une position hybride entre le naturel et l'ingénierie (Thompson, 2012, p. 14). Ce faisant, « les paysagistes intègrent les sciences écologiques au centre même de la conception urbaine en les imbriquant avec les enjeux sociaux, culturels et infrastructurels " (Waldheim, 2006a). Le discours du Landscape Urbanism revendique l'architecture du paysage comme une solution opérationnelle aux avancées théoriques recommandant une plus grande intégration écologique en urbanisme.

\section{Le Landscape Urbanism, discours de légitimation de l'expertise paysagiste renforçant des pratiques existantes : un savoir professionnel}

20 Certains auteurs voient le Landscape Urbanism comme une relecture de pratiques existantes: "nothing new, just landscape architecture or landscape planning represented" (Thompson, 2012, p. 8). Le Landscape Urbanism a permis de théoriser de manière évidente les pratiques paysagistes aux États-Unis. Il conforte des pratiques existantes et agit moins comme un facteur de renouvellement des pratiques que comme un facteur de conservation d'une démarche du projet de paysage structurée sur un discours professionnel. Ce discours ne fait que reformuler et réorganiser des idées, des concepts théoriques, et des pratiques paysagistes déjà existantes et révèle ainsi plus une "génération d'intellectuels professionnels " et un "mouvement professionnel » (Lindholm, 2011) que de réelles pratiques émergentes. Il entre dans le registre des théories instrumentales, décrivant et contrôlant les actions pratiques. Interprété comme un discours promotionnel en faveur des professions du paysage, il a sa place dans une société américaine organisée par des marchés essentiellement privés qui imposent aux paysagistes américains de mieux théoriser pour défendre leur profession, pour se vendre. Il prend la forme d'un discours de vente, dans le but d'acquérir de nouveaux marchés. Il faut donc comprendre le Landscape Urbanism comme un outil de promotion de la filière en vue de gagner des marchés.

21 Cette théorisation a été moins évidente en France bien que les pratiques soient tout à fait similaires. Les théorisations françaises et le discours des paysagistes français montrent de grandes similarités avec le Landscape Urbanism, par exemple dans le contexte du domaine de recherche du projet de paysage (Berque, Conan, Donadieu, Lassus et Roger, 1999 ; Besse, 2000). Pierre Donadieu (2006) considère que les pratiques professionnelles désignées par le Landscape Urbanism ont émergé en France et en 
Europe dans les années 1970, à travers les projets de Bernard Lassus, Michel Corajoud, Gilles Clément et plus récemment le travail du paysagiste Michel Desvigne (2009).

Nous venons donc de voir que la nature du discours du Landscape Urbanism fait partie du registre des discours professionnels, et a pour but de promouvoir la profession du paysage en s'appuyant sur le tournant écologique en urbanisme.

\section{Landscape Urbanism : un référentiel pour les pratiques des paysagistes contemporains?}

Dans cette seconde section, nous analysons dans quelle mesure il existe un lien entre les piliers du Landscape Urbanism tels qu'énoncés par Corner (2006) et les théories et pratiques des paysagistes en France. La mise en perspective du Landscape Urbanism par rapport au cas français permet de faire un constat de l'état des mouvements de théorisation en paysage en France. Ce lien n'assume pas une influence directe entre les deux corpus américain et français, mais plutôt une évolution similaire en parallèle. Nous organisons notre analyse autour de deux principes de classification des pratiques de conception de Corner dans le Landscape Urbanism Reader: "Les processus temporels » et « la stratification des surfaces» (Corner, 2006). Aussi, nous analysons la nature du Landscape Urbanism comme référentiel possible de pratiques paysagistes.

\section{«Processus de temps » : une approche dynamique des processus écologiques}

« Dynamique », « imprévisible », « en mouvement » sont autant d'adjectifs qualifiants les processus écologiques. Le Landscape Urbanism implique une reconnaissance de la nature comme environnement dynamique et relationnel et considère les échelles de temps dans le processus de conception comme alliant tous les agents du champ urbain dans un réseau continu d'interrelations (Corner, 2006). Le Landscape Urbanism cherche moins à étudier les objets que les processus (Thompson, 2012, p.12). Les théories produites par les professionnels français convergent dans la même direction.

Michel Desvigne (2009), face aux aléas incontrôlables des éléments naturels, défend l'idée d'un projet agissant sur les mécanismes de transformation des paysages, privilégiant les processus dynamiques plutôt que les plans masses fixes (Desvigne, 2002). Il apprécie le «jeu du temps, les stades successifs, la mise en valeur d'états jeunes, la coexistence de différents stades de développement qui concentrent, miniaturisent en une période courte, des mécanismes aux rythmes historiques" (Desvigne, 2009, p.12). La ville est donc à l'image de la nature: elle change progressivement, dans une "lenteur obligée ", comme un organisme vivant. Par exemple, à l'île Seguin, les jardins qu'il crée préfigurent le futur jardin et le premier espace public de l'île. 
Figure 1. Michel Desvigne Paysagiste, île Seguin, Paris (2012)

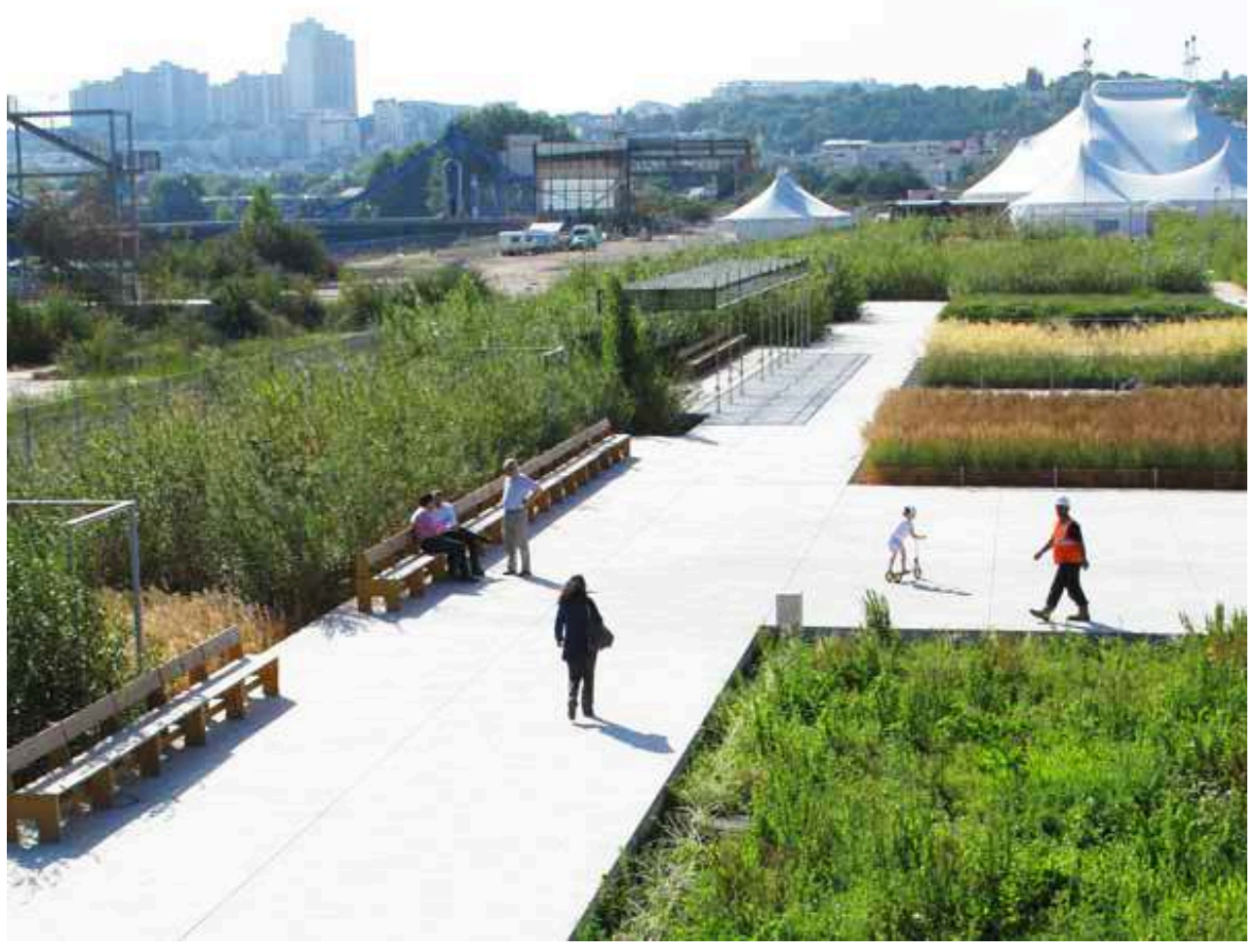

Source : Michel Desvigne Paysagiste.

Selon le paysagiste François-Xavier Mousquet (2011), l'approche écologique en paysage permet d'appréhender les espaces en constante transformation. Afin de traiter les sites contaminés des terrils dans le nord de la France, il laisse la flore se développer spontanément et observe une transformation progressive du paysage, grâce à une série de petits jardins carrés, « îlots de colonisation ». 
Figure 2. François-Xavier Mousquet, Agence Paysages, jardin du terril d'Haillicourt

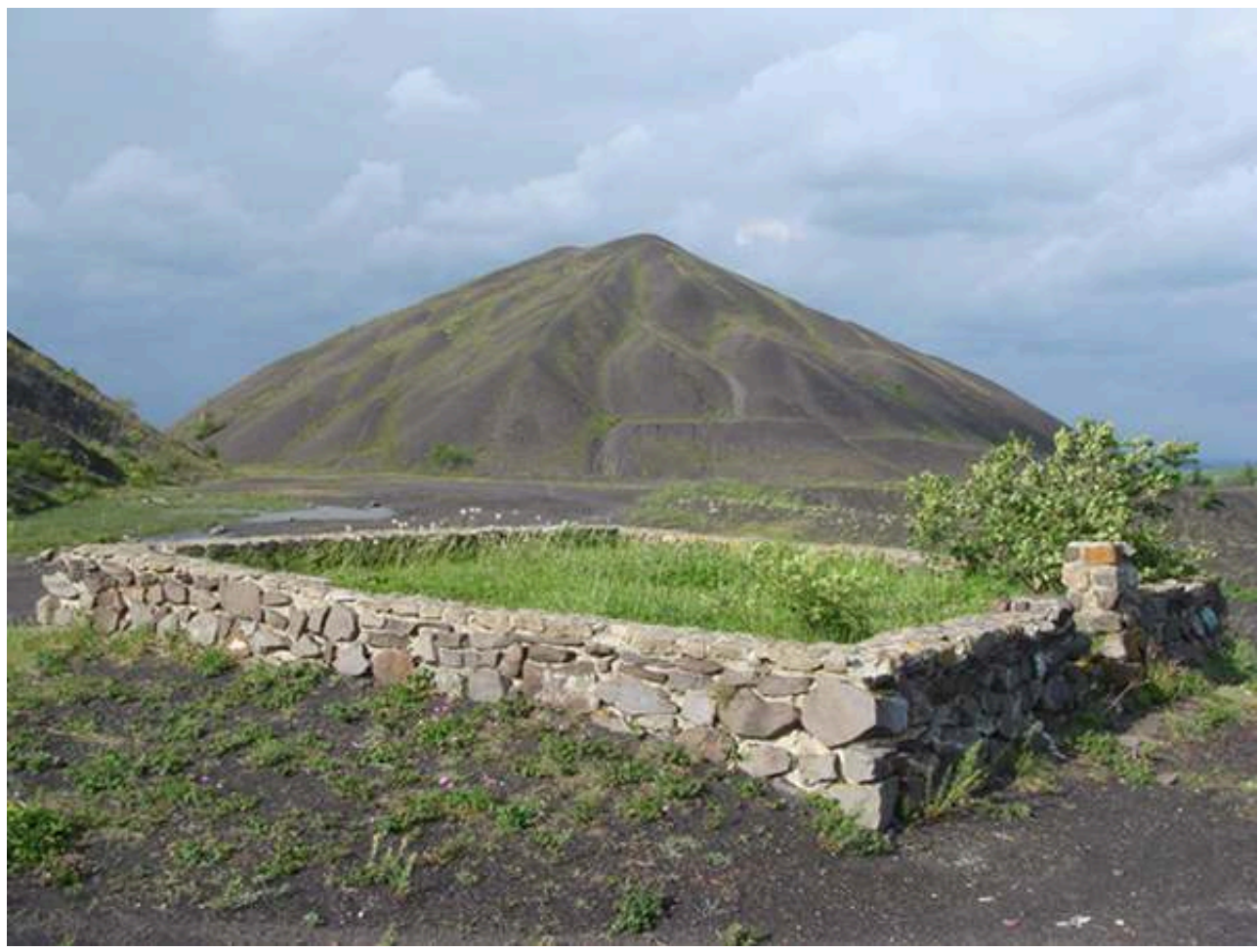

Source : Agence Paysages (2004).

Figure 3. François-Xavier Mousquet, Agence Paysages, jardin du terril d'Haillicourt

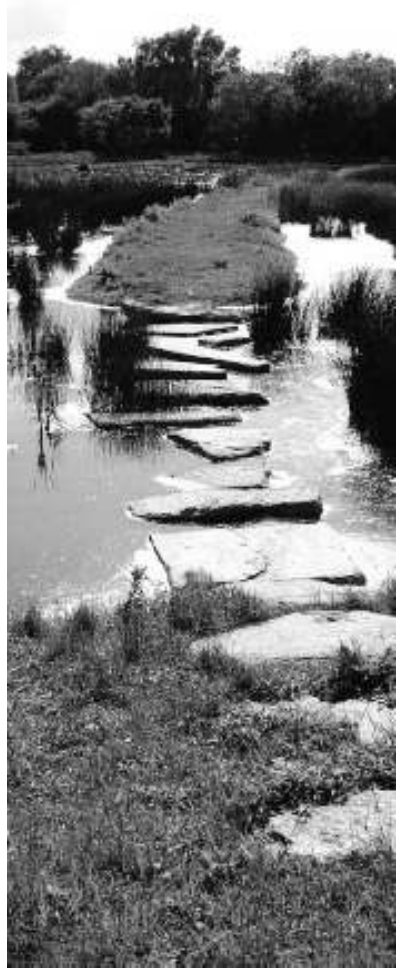

Source : Agence Paysages (2004). 

processus temporels. Dans sa théorie du tiers-paysage, « le fragment indécidé du Jardin planétaire désigne la somme des espaces où l'homme abandonne l'évolution du paysage à la seule nature » (Clément, 2004). À travers son approche du brassage planétaire, il examine «le résultat d'une agitation incessante des flux autour de la planète » (Clément, 2006). Dans une approche théorique similaire, le jardin en mouvement se trouve soumis à l'évolution résultant de l'interaction des êtres vivants dans le temps. Le jardinier cherche à utiliser les énergies en présence qu'il tente d'infléchir.

Figure 4. Gilles Clément, jardin de l'École normale supérieure de lettres de Lyon (2008)

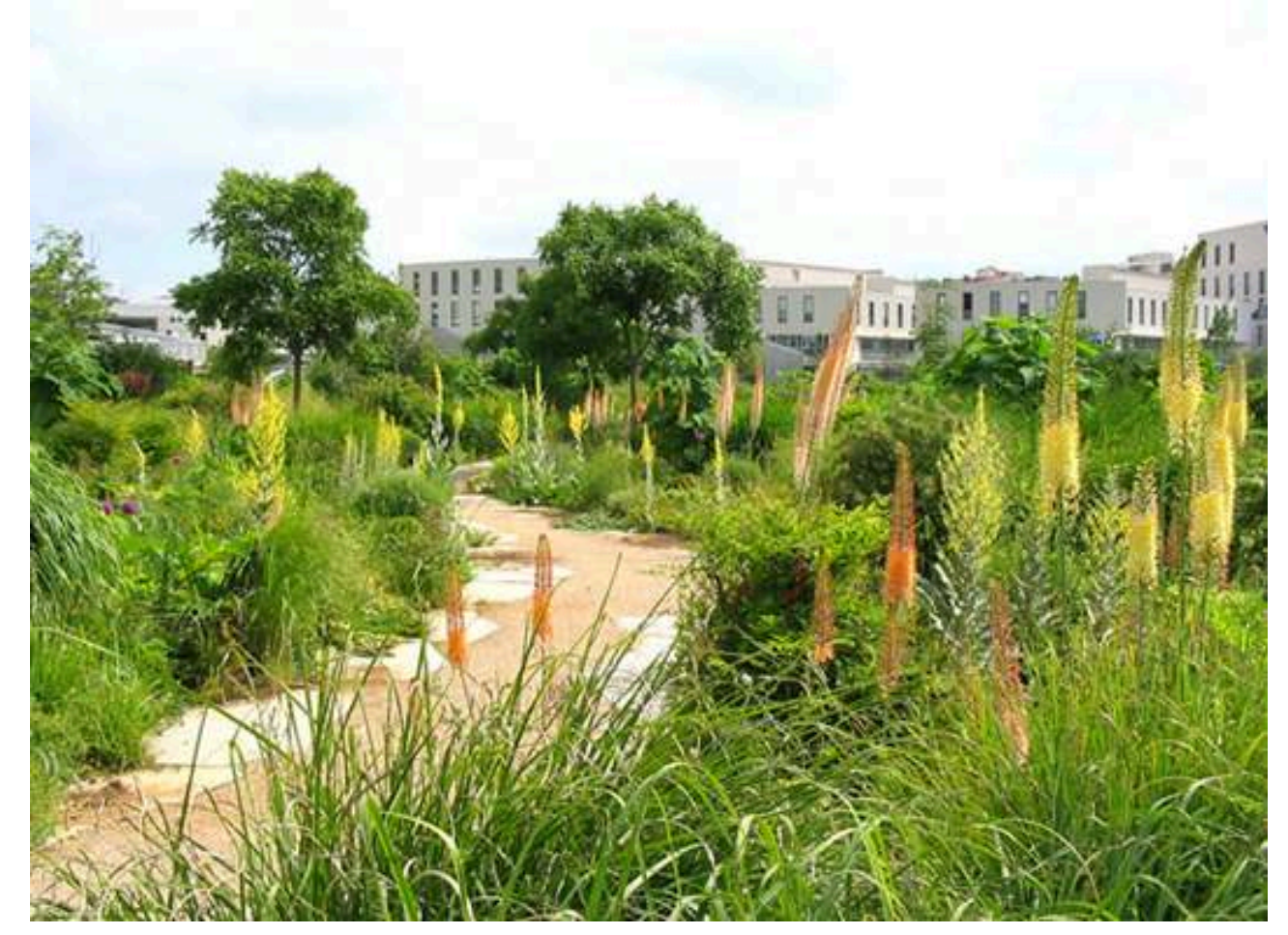

Source : Gilles Clément.

Il paraît donc évident que les principes de temporalité présentés par le Landscape Urbanism sont similaires à ceux des paysagistes français et ne témoignent pas d'une évolution marquante des pratiques paysagistes.

\section{"Superposition des surfaces » : une articulation des surfaces et des échelles pour une meilleure compréhension du site}

Dans le Landscape Urbanism, il y a l'idée que l'unité architecturale ne peut plus traduire la complexité des territoires à grande échelle. Le paysage devient une manière de saisir l'échelle de la ville diffuse à travers une approche systémique des continuités et des réseaux. Ainsi, le courant du Landscape Urbanism théorise la variation d'échelles spatiales. Afin de considérer divers systèmes, interrelations et réseaux existants, une 
superposition des surfaces est proposée comme moyen pour les paysagistes de considérer l'espace d'intervention. Cette superposition est héritée de l'approche McHargienne qui utilisait cette technique pour découper le site et son contexte en plans d'analyse de projet. Cependant, le Landscape Urbanism propose un pas méthodologique : il adapte cette approche et l'applique à une équipe pluridisciplinaire et à des connaissances scientifiques plus opérationnalisées pour le projet de paysage. Le processus permet de confronter les informations, le paysagiste peut commencer à comprendre les réseaux écologiques existant sur le site et au-delà des limites du site (Field Operations, 2006).

Figure 5. James Corner, Field Operations (2006)

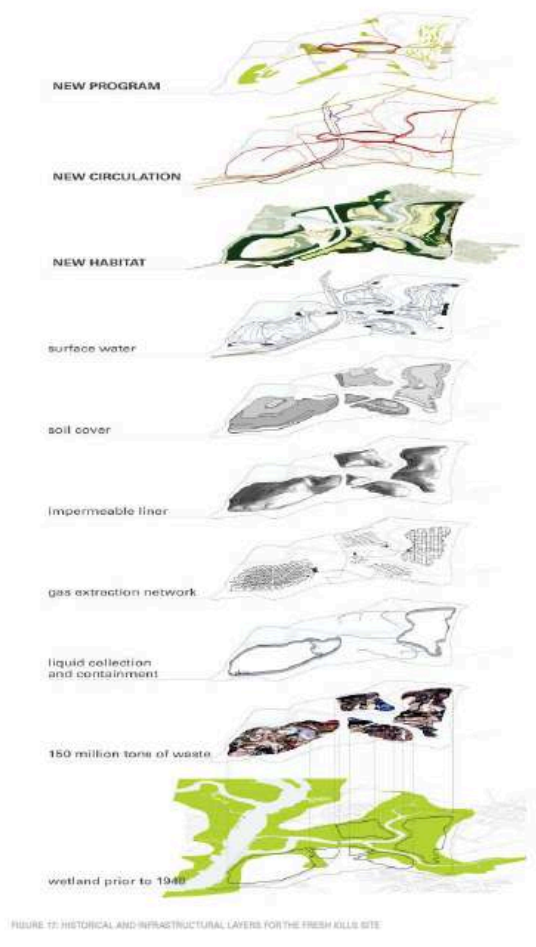

Source : James Corner Field Operations.

Ce sujet est par ailleurs abordé par les géographes et philosophes français travaillant sur les pratiques paysagistes (Cauquelin, 2000 ; Davodeau, 2011; Lussault, 2007). En décrivant la "surface de territoire comme une matrice active dotée d'une capacité de simulation", le philosophe français Sébastien Marot (2010, p.130) appelle à une «subversion de l'urbanisme appliquée à ménager des matrices territoriales plus complexes, plus profondes, capables d'articuler et de superposer davantage de plans dans la mémoire stratifiée des sites ", que le paysagiste français Corajoud (2010) intitule la "mémoire des lieux ». De ce fait, il nomme "suburbanisme» cette approche particulière de l'urbanisme pratiquée par les paysagistes qui jouent sur les stratifications des surfaces pour donner un ancrage à l'urbain diffus contemporain. La plus évidente illustration de cette approche spatiale du Landscape Urbanism à la française est aujourd'hui celle de Michel Desvigne. Il articule les différentes échelles dans ses projets et recompose les territoires urbains à travers leur géographie. C'est «l'amplification géographique» (Desvigne, 2009) qui utilise les formes naturelles 
existantes pour les renforcer. À Saclay, il crée un lien cohérent entre les espaces par une série de parcs.

Figure 6. Michel Desvigne Paysagiste, plateau de Saclay, Paris (2012)

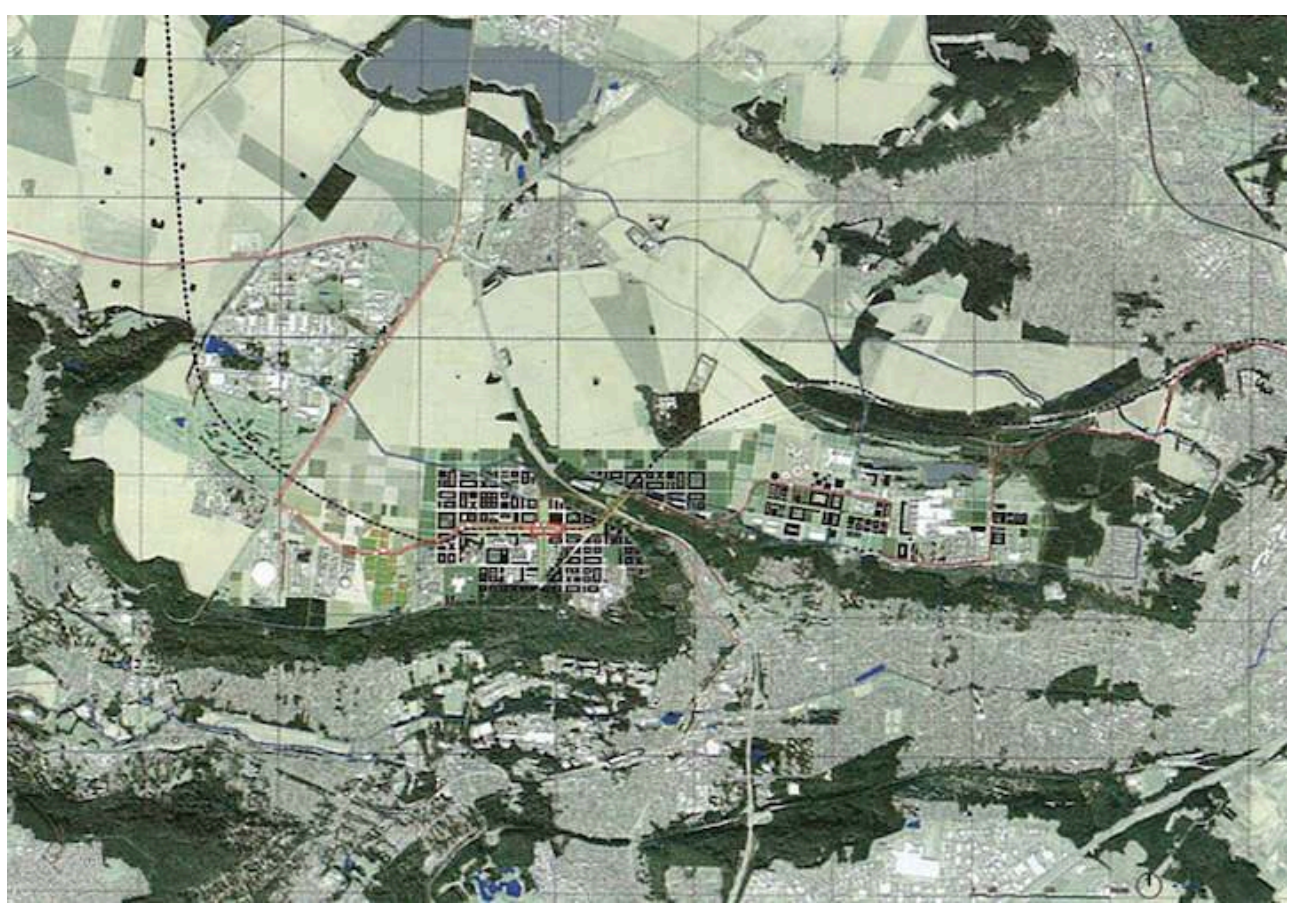

Source : Michel Desvigne Paysagiste

Le projet de Lyon Confluence illustre aussi cette idée et constitue une série de parcs le long de la Saône, avec des ramifications innervant l'île. 
Figure 7. Michel Desvigne Paysagiste, parc de Saône, Lyon Confluence (2009)

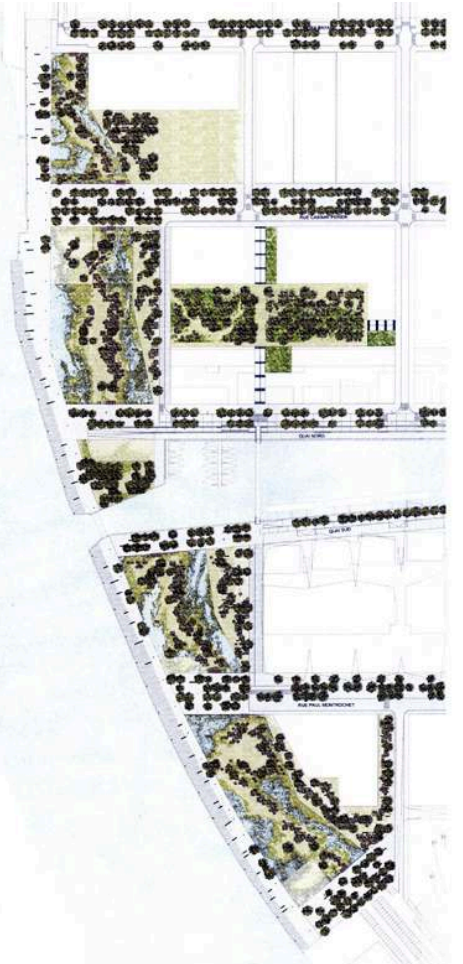

Source : Michel Desvigne Paysagiste

Figure 8. Michel Desvigne Paysagiste, parc de Saône, Lyon Confluence (2009)

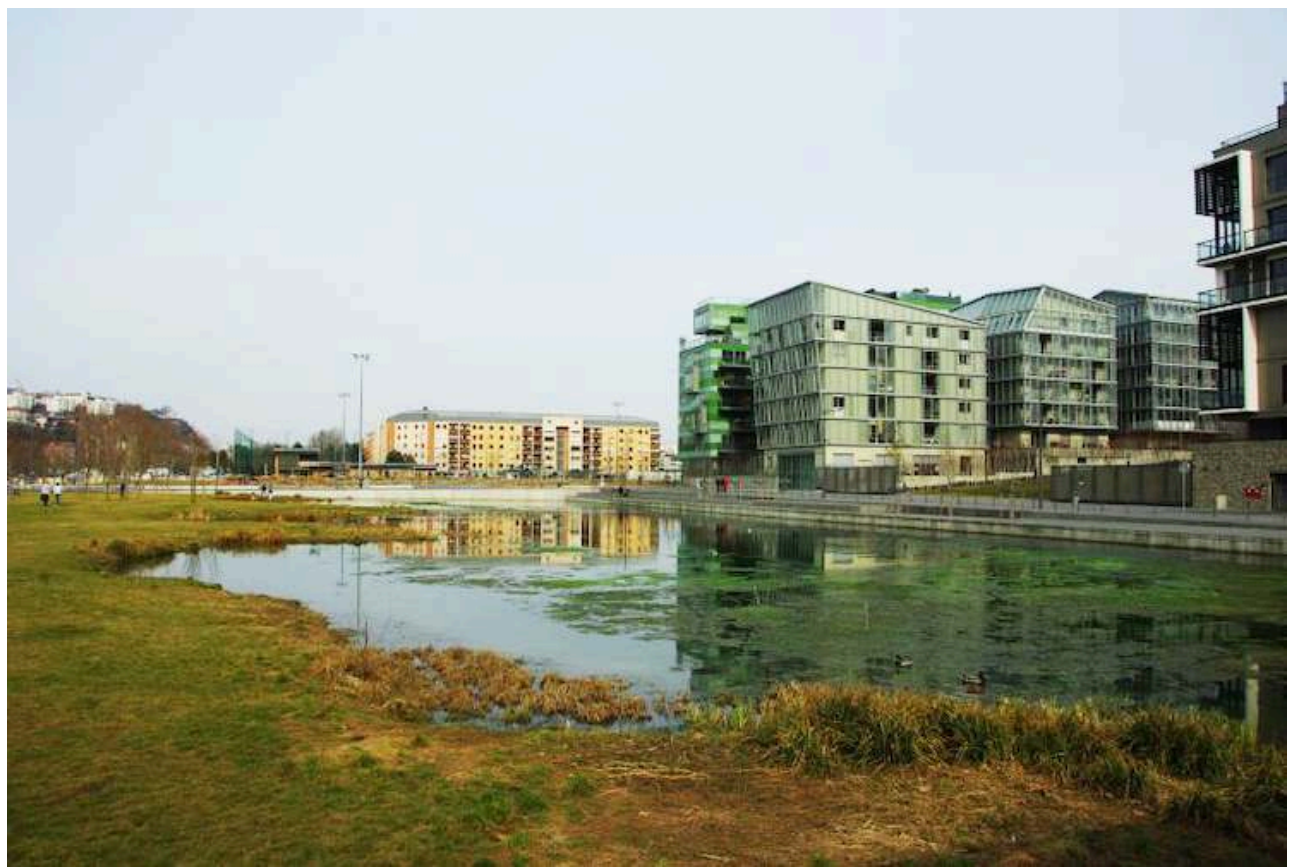

Source : Michel Desvigne Paysagiste.

Plus spécifiquement, cette question des échelles amène à réfléchir sur la compréhension du site. Un projet de paysage s'applique sur un site mais doit prendre 
en compte des échelles qui le dépassent. Dans l'ouvrage Landscape Urbanism Reader, Czerniak (2006) aborde l'impact significatif du projet "sur le site et au-delà du site ", alors que Pollak (2006) parle de «traiter des zones bien plus grandes» que le site. Leur clé d'entrée s'appuie sur la nécessité d'étudier les continuités écologiques au-delà des limites du site. Burns et Kahn (2005), en décrivant la dynamique du site au-delà de son contour, définissent la zone de contrôle, la zone d'influence et la zone d'effet.

Il existe en France des théories similaires sur ce sujet. Le paysagiste ne travaille pas seulement à l'intérieur des limites de son site. La notion de paysage s'accommode mal des limites fixes: « Il n'y a pas de contours francs, chaque surface tremble et s'organise de telle manière qu'elle ouvre essentiellement sur le dehors" (Corajoud, 2010). Le concepteur se détache ainsi de son site et s'ouvre à un contexte plus large. Sébastien Marot (2010) évoque une approche du site qui serait très spécifique au paysagiste, Hervé Davodeau (2008) décrit le socle géographique naturel, permanent, spécifique à partir duquel les paysagistes développent le projet de paysage.

Dans cette section, les similarités entre le Landscape Urbanism et les discours paysagistes français sont grandes. Le Landscape Urbanism utilise une sémantique empruntée à l'écologie pour reformuler des concepts et renforcer des pratiques déjà existantes. En constituant un référentiel de pratiques, le Landscape Urbanism incarne un savoir explicite, faisant office de modèle pour le reste de la profession, et constitue une théorie instrumentale qui décrit et contrôle les actions pratiques.

\section{Le Landscape Urbanism : validation par la recherche et transmission par l'enseignement}

Nous avons montré que le statut du discours du Landscape Urbanism serait plutôt de nature explicite, établissant des principes de classification des pratiques paysagistes, constituant un référentiel. C'est aussi un discours professionnel, émanant de la pratique, légitimant une expertise, provenant de l'argumentation du projet. Dans cette dernière section, nous étudions le statut du Landscape Urbanism en tant que discours systématique, influençant la recherche, mais aussi discours pédagogique, transmissible aux futurs paysagistes.

\section{Théorisation des pratiques paysagistes et recherche, le renforcement du paysage comme discipline : vers un savoir systématique?}

37 Le Landscape Urbanism se veut être « un solvant qui estompe les frontières entre les disciplines " (Thompson, 2012, p. 10) et une "convergence de distinctions professionnelles et institutionnelles vers une nouvelle pratique plus intégrative " (Corner, 2006). C'est donc un discours méthodologique sur la collaboration professionnelle, contribuant à la pluridisciplinarité. De la même manière, les discours théoriques français promeuvent " un décloisonnement des expertises techniques et un positionnement de l'aménagement dans une réalité pluri-professionnelle » (Jannière et Pousin, 2007). Pour autant le Landscape Urbanism propose une position paradoxale : d'un côté elle prône cette collaboration des disciplines nécessaire en aménagement (Lindholm, 2011) mais, d'un autre, elle promeut le paysagisme comme seule discipline 
capable d'utiliser l'écologie appliquée à une stratégie d'urbanisme. C'est là toute l'ambiguïté du Landscape Urbanism. S'agit-il d'une translation des professions de l'urbanisme afin d'utiliser le paysage comme clé d'entrée du projet urbain?

Pourtant, ce n'est que lorsque le paysage sera identifié comme une discipline à part entière qu'il participera pleinement au phénomène pluridisciplinaire observé en urbanisme (Ramadier, 2004). Le paysage est peu reconnu comme discipline académique (Deming et Swaffield, 2011). En France, peu de paysagistes réalisent des thèses. Ce problème institutionnel inhibe la recherche en paysage et le développement de la profession. D'où la nécessité grandissante de renforcer l'identité disciplinaire du paysage, avec des méthodologies de recherche et une épistémologie propre. Ainsi, une demande de recherche paysagiste émerge. Des initiatives comme le développement d'une théorisation dans la revue Projets de paysage, la création d'un master théorie et démarches de projet de paysage ou l'enseignement sur l'histoire des pratiques paysagistes cherchent à y répondre.

La théorisation des pratiques paysagistes, telle que le discours du Landscape Urbanism, peut renforcer le paysage comme domaine disciplinaire et créer un lien entre pratiques et recherche. Cependant, la question d'introduire un discours professionnel comme le Landscape Urbanism dans le monde académique peut représenter un danger. Du fait des caractéristiques appliquées que prennent les activités de conception, l'utilisation du mot « recherche » peut parfois être équivoque dans la théorie du projet de paysage. La recherche implique des données, une méthodologie et la validation d'hypothèses. Cependant, dans les disciplines appliquées comme le paysage, la recherche base ses questions sur des problèmes rencontrés par des professionnels dans le champ de la pratique. Il est parfois difficile d'identifier la limite entre recherche et pratique. Les paysagistes-concepteurs ont tendance à développer une rhétorique publicitaire sur leurs projets et pratiques : c'est le pouvoir du discours technocratique à travers lequel l'expert se légitime (Foucault, 1991). Le chercheur britannique Thompson (2012) met en évidence le fait qu'«utiliser le jargon de l'expert plutôt que le langage quotidien permet de garder le pouvoir et le contrôle dans les mains des technocrates ». Le monde académique se doit de déceler ces rhétoriques et de discuter l'intégrité des discours professionnels afin d'éviter les dangers du corporatisme et de l'autopromotion. Certes le Landscape Urbanism peut être utile pour renforcer le paysage en tant que discipline, mais il ne constitue pas pour autant une théorie de recherche.

\section{Une meilleure intégration des enjeux écologiques dans la pédagogie du paysage : un savoir transmissible}

Les paysagistes s'inscrivent dans une pratique professionnelle. Porteurs d'une tradition et d'une culture du paysagisme, ils sont les héritiers de leurs aînés. Chacun d'entre eux appartient donc à une école de pensée, qui transmet des modèles et des références, des systèmes de pensées paysagistes, théoriques et pratiques (Toublanc, 2010). La mise en pratique de l'écologie urbaine et de l'écologie du paysage passe par l'enseignement et la pédagogie de ces disciplines dans les écoles de paysage.

41 À l'École nationale supérieure du paysage de Versailles, « la place que tient l'écologie du paysage dans la pédagogie est peu lisible et la discipline n'est pas enseignée en tant que telle. Son enseignement est adapté au projet de paysage et croise les démarches cognitivies et sensibles» (Rumelhart, 2010). Cela passe par une «meilleure articulation 
entre connaissances des processus (naturels et sociaux) et action, le projet de paysage. Mais l'écologie est désormais considérée comme une science appliquée. Cela est donc à l'origine d'innovations pédagogiques mais aussi de tensions au sein de la communautés des enseignants, composées de praticiens et d'universitaires. Il y a une difficulté dans la formation des paysagistes à combiner la question des représentations sociales, avec une démarche et une pratique de projet, et à l'inscrire dans une logique d'action » (Toublanc, 2010). Ainsi le Landscape Urbanism, par une promotion de l'opérationnalisation des principes écologiques à travers le projet de paysage, pourrait incarner une école de pensée. Cependant, ses applications dans les programmes pédagogiques sont encore rares, et son enseignement en Europe est détenu par les architectes et se concentre à l'école de la Architectural Association à Londres, qui offre un master of Art en Landscape Urbanism.

\section{Conclusion : Landscape Urbanism, vers une théorie critique?}

L'objectif principal de cet article était de comprendre si la théorie du Landscape Urbanism constitue un facteur d'évolution des pratiques paysagistes de conception. Nous avons étudié la fonction, le statut et la nature du discours paysagiste à travers une analyse interprétative du Landscape Urbanism. Nous avons établi dans un premier temps que ce mouvement américain n'est pas un facteur d'évolution des pratiques mais constitue un discours professionnel de nature descriptive et opérationnelle. En tant que savoir explicite, il décrit, classe les pratiques paysagistes et fait office de référence dans la profession. Ce discours professionnel émane de la pratique, provient de l'argumentation du projet et légitime l'expertise paysagiste. Ce discours paysagiste exploite l'actuel tournant écologique en urbanisme pour promouvoir les professions $\mathrm{du}$ paysage. C'est aussi un discours sur la collaboration avec les autres professions, la pluridisciplinarité. Le Landscape Urbanism aurait vocation à devenir un discours pédagogique, voire une école de pensée, servant à transmettre ce discours modèle aux futurs paysagistes, dans le cas où ses principes sur l'opérationnalisation de l'écologie à travers le projet de paysage seraient enseignés plus largement dans les écoles de paysage. Certes le Landscape Urbanism peut être utile pour renforcer le paysage en tant que discipline, mais il ne constitue pas pour autant une théorie de recherche. Faute d'être validé par la communauté scientifique, il renforce tout au plus la discipline du paysage. Le Landscape Urbanism pourra-t-il donc à terme acquérir le statut de discours critique au sein de sa communauté professionnelle, capable d'en provoquer une mutation, un changement vers de nouvelles pratiques professionnelles innovantes?

Je remercie mon codirecteur de thèse Hervé Davodeau de l'École du paysage d'Angers (Agrocampus Ouest) pour ses conseils, relectures et corrections, ainsi que le département de paysage de la Swedish University of Agricultural Sciences SLU pour son accueil dans le cadre du séminaire doctoral sur « Landscape Urbanism - Landscape Analysis » à Alnarp à Malmö en Suède en septembre 2011. 


\section{BIBLIOGRAPHIE}

Alberti, M., Advances in Urban Ecology, New York, Springer Science, 2008.

Amossy, R., «Argumentation et Analyse du discours : perspectives théoriques et découpages disciplinaires ", dans Argumentation et analyse du discours : perspectives théoriques et découpages disciplinaires, mis en ligne le 6 septembre 2008, URL : http://aad.revues.org/200.

Berque, A., Médiance de milieux en paysages, Paris, Belin, 2000.

Berque, A., Conan, M., Donadieu, P., Lassus, B., et Roger, A., Mouvance. Cinquante mots pour le paysage, Paris, Éditions de la Villette, 1999.

Bertrand, M., Blot, F., Dascon, J., Gambino, M., Milian, J., et Molina, G., « Géographie et représentations : de la nécessité des méthodes qualitatives », Recherches qualitatives. Bilan et prospectives de la recherche qualitative en sciences humaines et sociales, coll. hors série « Les Actes ", $n^{\circ} 3,2007$.

Besse, J.-M., Voir la terre. Six essais sur le paysage et la géographie, Arles, Actes Sud, 2000.

Brundtland, G. H., « Report of the World Commission on Environment and Development », United Nations, General Assembly Resolution, 1987.

Burel, F. et Baudry, J., Écologie du paysage. Concepts, méthodes et applications, Paris, TEC \& DOC, 1999.

Burns, C. et Kahn, A., Site matters. Design concepts, Histories and Strategies, New York and London, Routledge, 2005.

Brown, G. et Yule, G., Discourse analysis, Cambridge, Cambridge University Press, 1983.

Cauquelin, A., L'Invention du paysage, Paris, PUF, 2000.

Clément, G., Le Jardin en mouvement. De la Vallée au jardin planétaire, Paris, Sens \& Tonka, 2006.

Clément, G., Manifeste pour le Tiers paysage, Paris, Sujet-Objet, 2004.

Corajoud, M., Le Paysage, c'est l'endroit où le ciel et la terre se touchent, Arles/Versailles, Acte Sud/ École nationale supérieure du paysage de Versailles-Marseille, 2010.

Cormier, L., " Les trames vertes : entre discours et matérialités, quelles réalités ? ", thèse, Agrocampus Ouest, université d'Angers, 2011.

Corner, J., "Terra fluxus », dans Waldheim, C. (ed.), The Landscape Urbanism Reader, New York, Princeton Architectural Press, 2006, p. 21-33.

Corner, J., «A discourse on theory I : Sounding the depths-origins, theory and representation ", Landscape Journal, vol. 17, $\mathrm{n}^{\circ}$ 2, 1990, p. 61-78.

Czerniak, J., « Looking back at Landscape Urbanism : Speculations on Site », dans Waldheim, C. (ed.), The Landscape Urbanism Reader, New York, Princeton Architectural Press, 2006, p. 105-123.

Davodeau, H., «La dimension spatiale de l'action paysagère ", Annales de géographie, vol. 3, 2011, p. 246-265.

Davodeau, H., « "Le socle”, matériau du projet de paysage », Projets de paysage, n 1 , publié en décembre 2008, URL : http://www.projetsdepaysage.fr/fr/

le_socle_materiau_du_projet_de_paysage. 
Deleuze, G. et Guattari, F., Capitalisme et schizophrénie 2. Mille plateaux, Paris, Éditions de Minuit, 1980.

Deming, E. et Swaffield, S., Landscape Architectural Research : Inquiry, Strategy, Design, New York, John Wiley \& Sons, 2011.

Descola, P., Par-delà nature et culture, Paris, Gallimard, 2005.

Desvigne, M., Intermediate Natures : The Landscapes of Michel Desvigne, Basel, Boston, Berlin, Birkhaüser Verlag AG, 2009.

Desvigne, M., « Penser la ville par le paysage », dans Masboungi, A. (dir.), Penser la ville par le paysage, Paris, La Villette, 2002.

Donadieu, P., « Landscape Urbanism in Europe : from brownfields to sustainable urban development ", Journal of Landscape Architecture JoLA, vol. 2, nº 2, 2006.

Donadieu, P. et Périgord, M., Clés pour le paysage, Paris, Ophrys, 2005.

Ducrot, O., " Argumentation rhétorique et argumentation linguistique », dans Doury, M. et Moirand, S. (dir.), L'Argumentation aujourd'hui. Positions théoriques en confrontation, Paris, Presses de la Sorbonne nouvelle, 2004.

Eisen, J. B., « Brownfields policies for sustainable cities », Duke Environmental Law and Policy Forum, vol. 9, 1999, p. 188-229.

Emelianoff, C., « Urbanisme durable ?» Écologie et politique, vol. 29, 2004, p. 15-19.

Field Operations, Fresh Kills Park : Lifescape, Draft Masterplan, New York, New York City Department of city Planning, 2006.

Forman, R., et Gordon, M., Landscape Ecology, New York, John Wiley and Sons, 1986.

Foucault, M., « On power », dans Dovey, K. (ed.), Framing Places. Mediating power in built form, London \& New York, Routledge, 1991.

Fry, G., « Multifunctional landscapes - towards transdisciplinary research », Landscape and Urban Planning, vol. 57, issue 3-4, 2001, p. 159-168.

Gaston, K. J., « Urban Ecology » dans Gaston, K. J. (ed.), Urban Ecology, Cambridge, Cambridge University Press, 2010.

Jannière, H. et Pousin, F., " Paysage urbain : d'une thématique à un objet de recherche ", Strates. Paysage urbain : genèse, représentations, enjeux contemporains, 2007, vol. 13 ; mis en ligne en octobre 2008, URL : http://strates.revues.org/4953.

Latour, B., Politiques de la nature. Comment faire entrer les sciences en démocratie, Paris, Editions la Découverte, 1999.

Lindholm, G., «"Visible gestures" : on urban landscape perspectives in planning ", Planning Theory, London, Sage, vol. 11, 2012.

Lussault, M., L'Homme spatial, Paris, Éditions du Seuil, 2007.

McHarg, I., Design with Nature, coll. « Wiley Series in Sustainable Design », New York, John Wiley and Sons, 1969.

Maingueneau, D., «L'analyse du discours et ses frontières », Marges linguistiques, vol. 9, 2005,

Marcuse, P., "Sustainability is Not Enough », Environment and Urbanization, vol. 10, 1998, p. 103-112. 
Marot, S., L'Art de la mémoire, le territoire et l'architecture, Paris, Éditions de la Vilette, 2010.

Mousquet, F.-X., « Paysage et environnement : une poétique commune », Espaces et sociétés, vol. 146, $\mathrm{n}^{\circ} 3,2011$.

Nassauer, J. I., « Landscape as medium and method for synthesis in urban ecological design », Landscape and Urban Planning, vol 106, 2012, p. 221-229.

Odum, E. P. et Barrett, G. W., Fundamentals of ecology (5e édition), St. Paul MN, Thomson Brooks/ Cole, 2005.

Paltridge, B., Discourse anaysis : an introduction, London, Continuum, 2007.

Pollak, L., « Constructed Ground : Questions of scales », dans Waldheim, C. (ed.), The Landscape Urbanism Reader, New York, Princeton Architectural Press, 2006, p. 125-139.

Ramadier, T., « Transdisciplinarity and its challenges : the case of urban studies », Futures, vol. 36, in $^{\circ} 4,2004$, p. 423-439.

Reimer, M. H., « Unsettling eco-scapes : aesthetic performances for sustainable futures ", Journal of Landscape Architecture, vol. 1, 2010, p. 24-37.

Repetti, A. et Desthieux, G., « A relational indicator set model for urban land-use planning and management : methodological approach and application in two case studies ", Landscape and Urban Planning, vol. 77, 2006, p. 196-215.

Roger, A., Court Traité du paysage, Paris, Gallimard, Coll. « Sciences humaines », 1997.

Rumelhart, M., « Comment rapprocher l'écologie du paysage et le projet de paysage ? », Les Carnets du paysage. Écologies à l'œuvre, vol. 19, 2010, p. 29-31.

Silverman, D., Doing qualitative research : a pratical handbook, London, Sage, 2005.

Snep, R. et Opdam, P., « Intergating nature values in urban panning and design », dans Gaston, K. J. (ed.), Urban Ecology, Cambridge University Press, 2010.

Steiner, F., « Landscape ecological urbanism : Origins and trajectories », Landscape and Urban Planning, vol. 100, $\mathrm{n}^{\circ} 1,2011$, p. 333-337.

Swaffield, S., Theory in landscape architecture, Philadelphia, University of Pennsylvania Press, 2002.

Thompson, I. H., « Ten Tenets and Six Questions for Landscape Urbanism », Landscape Research, vol. $1, \mathrm{n}^{\circ} 37,2012$, p. 7-26.

Toublanc, M., « Entre recherche et formation : le point de vue d'une sociologue », Les Carnets du paysage. Écologies à l'œuvre, vol. 19, 2010, p. 43-50.

Waldheim, C., «Introduction : a reference manifesto », dans Waldheim, C. (ed.), The Landscape Urbanism Reader, New York, Princeton Architectural Press, 2006a, p. 13-19.

Waldheim, C., «Landscape as Urbanism », dans Waldheim, C. (ed.), The Landscape Urbanism Reader, New York, Princeton Architectural Press, 2006b, p. 35-53.

\section{RÉSUMÉS}

Cet article examine l'influence que le Landscape Urbanism a sur le développement des pratiques professionnelles des paysagistes dans le contexte français. Il y a dix ans, Charles Waldheim développait cette théorie aux États-Unis, positionnant les paysagistes comme étant les plus à même d'organiser la ville par l'intégration des sciences écologiques au centre de la conception 
urbaine. Cet article utilise la méthodologie de l'analyse interprétative de discours et examine le Landscape Urbanism en lien avec les théories et discours paysagistes similaires dans le contexte français. Nous montrons que le Landscape Urbanism est un discours professionnel de référence pour la légitimation de l'expertise paysagiste en proposant une classification des pratiques de conception émergentes. Nous montrons ensuite qu'il renforce la discipline du paysage dans le domaine de la recherche et de l'enseignement. Le Landscape Urbanism pourra-t-il à terme acquérir le statut de théorie critique, capable de provoquer une mutation de la profession vers des pratiques innovantes?

This paper examines the influence that Landscape Urbanism has on the development of Landscape Architects' professional practices in the French context. Ten years ago, in the United States, Charles Waldheim developed the theory of Landscape Urbanism, which positions the landscape architect as being the most capable profession of organizing the city by integrating ecological sciences at the very centre of urban design. This paper proposes an interpretative discourse analysis on Landscape Urbanism in parallel with similar theories and discourses in the French sphere. We suggest that Landscape Urbanism is a professional discourse that legitimates the expertise of Landscape Architects, and proposes a classification of emergent landscape design practices. We then show that Landscape Urbanism strengthens the discipline of Landscape Architecture in education and academic research. Will Landscape Urbanism, in the long term, acquire the status of critical theory, capable of provoking a mutation of the profession towards more innovative practices?

\section{INDEX}

Keywords : landscape urbanism, landscape architect, ecology, discourse, theory, practices Mots-clés : landscape urbanism, paysagiste, écologie, discours, théories, pratiques

\section{AUTEUR}

\section{ANAIIS LEGER-SMITH}

Ingénieur paysagiste, Anaïs Leger-Smith est doctorante en troisième année aménagement et paysage à l'École du paysage d'Angers, Agocampus Ouest-université d'Angers. Elle a été membre invité dans l'équipe de recherche de l'École d'architecture, d'urbanisme et de paysage à Newcastle University en 2012 en Angleterre. Son doctorat porte sur l'évolution des pratiques paysagistes au regard des nouveaux enjeux écologiques.

f.anais[at]gmail[dot]com 